\title{
Massive stone burden in an ileocecal pouch: A preventable condition?
}

\author{
Anne Sophie Valiquette; Diego Barrieras, MD, FRCSC; Michael McCormack, MD, FRCSC
}

Department of Surgery, University of Montreal, Montreal, QC

Cite as: Can Urol Assoc J 2014;8(5-6):e358-60. http://dx.doi.org/10.5489/cuaj.1537 Published online May 21, 2014.

\section{Abstract}

The challenges of transition from the pediatric to the adult setting have been reported. We describe a case of massive stone burden in the ileocecal pouch of a patient lost to follow-up after his transfer from a pediatric to an adult institution. Although we successfully managed the patient and retrieved the pouch stones without any complications, several surgical complications may occur in a patient with a history of extensive abdominal surgery and bladder exstrophy. This patient's late complication might have been prevented with a more efficient transfer from a pediatric to an adult institution.

\section{Case report}

A 39-year-old male presented with symptoms of increasing difficulty in self-catheterization of a continent stoma. He denied any history of hematuria, pain or fever. He had a medical history of bladder exstrophy and he had underwent multiple intra-abdominal surgical interventions for this condition. During his late childhood, an ileocecal pouch was done at a pediatric hospital. The patient was followed diligently in the pediatric setting until he reached 18, at which time he was referred to an adult institution. However, the patient was lost to follow-up after his transfer.

The patient had been doing self-catheterizations of his continent stoma for many years, but he noticed an increasing difficulty in emptying his pouch. He also had to significantly increase the number of self-catheterizations per day.

A kidney, ureter, bladder (KUB) scan showed massive stone burden in his ileocecal pouch (Fig. 1). The patient's serum creatinine was $146 \mathrm{umol} / \mathrm{L}$. An abdominal computed tomography scan (Fig. 2) showed no stones in the upper urinary tract, although some stones were found in the ileal segment of the pouch. The patient underwent open surgery and all the stones were successfully removed. A biochemical stone analysis showed the stone composition as struvite (Fig. 3).

\section{Discussion}

Although ileocecal pouches are safe and effective, longterm complications do occur. ${ }^{1-4}$ Pouch stones have been described in about $10 \%$ of patients and risk factors include the use of metallic staples and an intussuscepted ileal nipple. Struvite stones form in alkaline urine from infection with a urea-splitting organism and these stones can be treated with minimally invasive surgical techniques if the stone burden is small. However, in our patient open surgery was required because of the massive stone burden. Although we successfully managed the patient and retrieved the pouch stones without any complications, several complications may occur in a patient with a history extensive abdominal surgery and bladder exstrophy. A laparotomy was a high-risk undertaking in this patient. Potential complications of open surgery are major and could have profoundly altered the patient's quality of life. A much safer approach would have involved an adequate follow-up after his transfer from the pediatric institution where he was initially treated.

This patient's long-term complication illustrates the problematic transfer from a pediatric to an adult institution. The challenges of transition from the pediatric to the adult setting have been described. ${ }^{5}$ Recommendations pertaining to the transfer include a coordinated effort to ensure a successful transition from a pediatric to an adult institution. The Canadian Pediatric Society (CPS) issued a policy statement in 2007 (and reaffirmed in 2013). ${ }^{6}$ The CPS supports the provision of developmentally appropriate care for youth with special healthcare needs as they move to an adult-oriented healthcare system. We believe this patient's condition illustrates the importance of this problem and could serve as an example of the need to apply these CPS recommendations. 


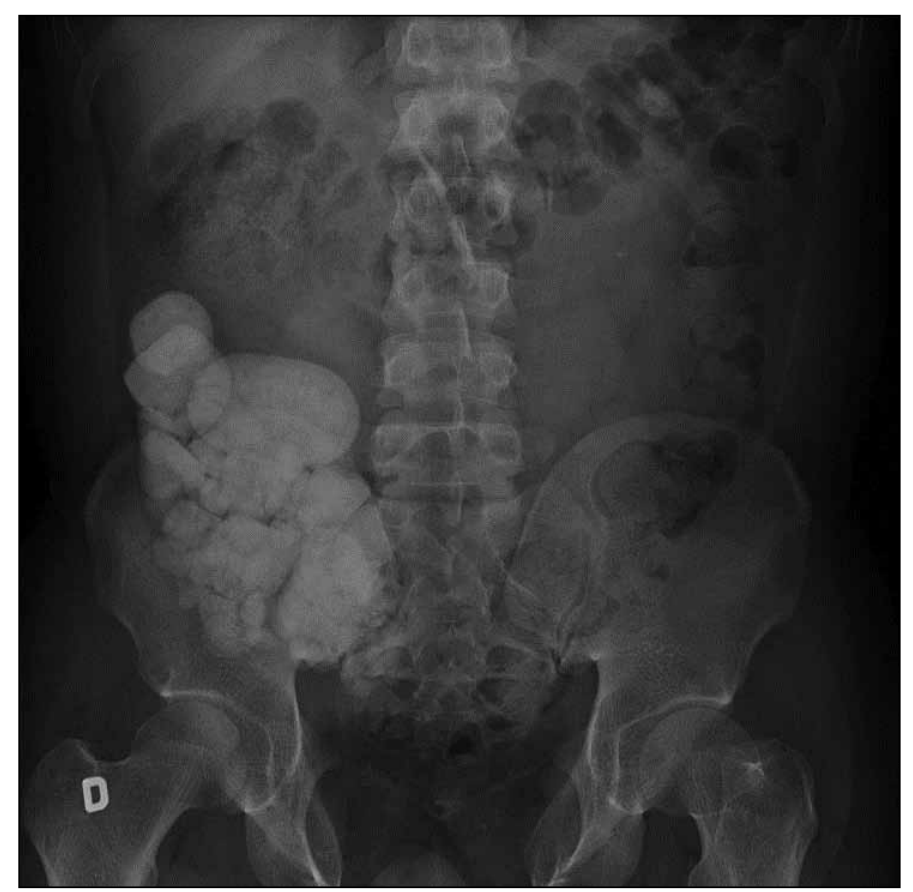

Fig. 1. Kidney, ureter, bladder (KUB) scan shows massive stone burden in ileocecal pouch.

\section{Conclusion}

Our case report describes a patient with massive stone burden in a continent ileocecal pouch. The patient had a history of bladder exstrophy and underwent an operation to create an ileocecal pouch during his childhood at a pediatric hospital. The patient was followed in a pediatric setting until he reached 18, at which time he was referred to an adult institution. However, he was lost to follow-up. Although open surgery was uneventful, a laparotomy was a high-risk undertaking for this patient. Potential complications of open

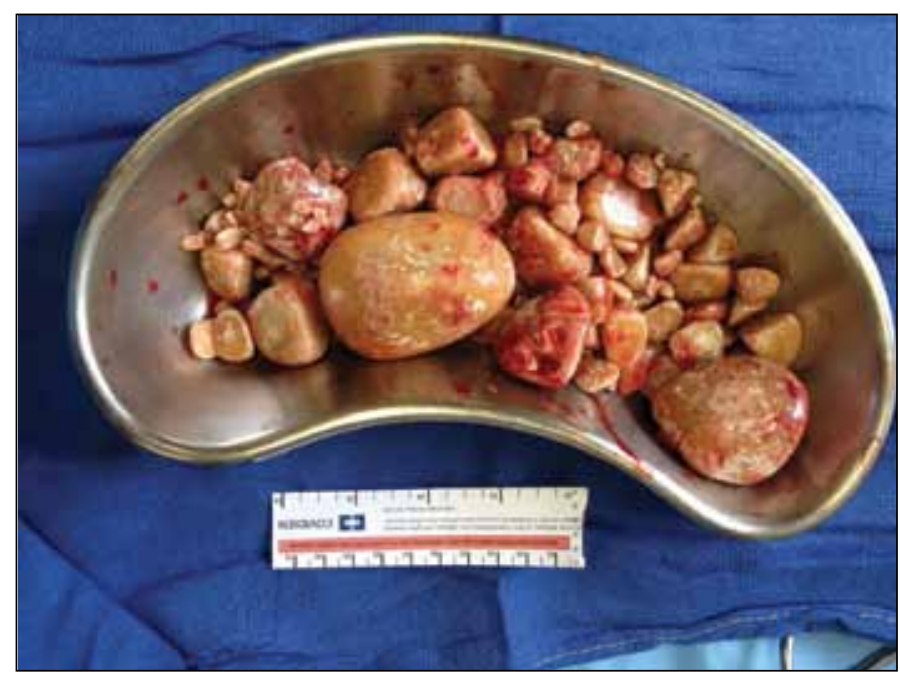

Fig. 3. Over 100 stones were removed from the pouch. Biochemical stone analysis showed the stone composition to be struvite.

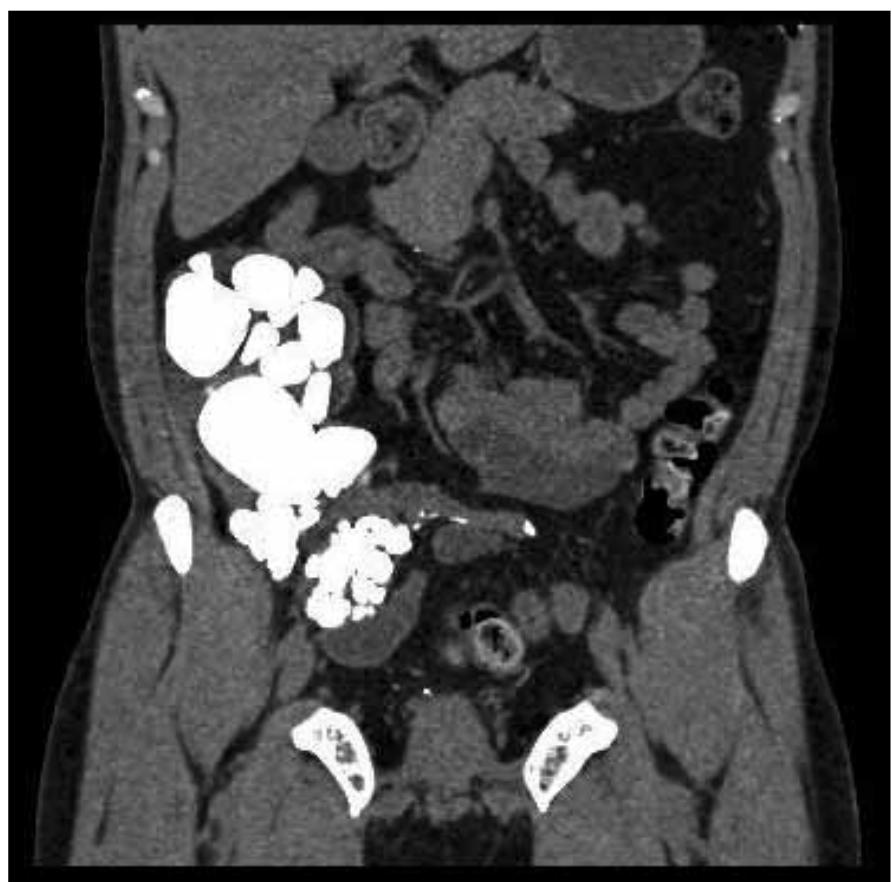

Fig. 2. A computed tomography scan shows massive stone burden in ileocecal pouch and a few calculi in the ileal part of the pouch.

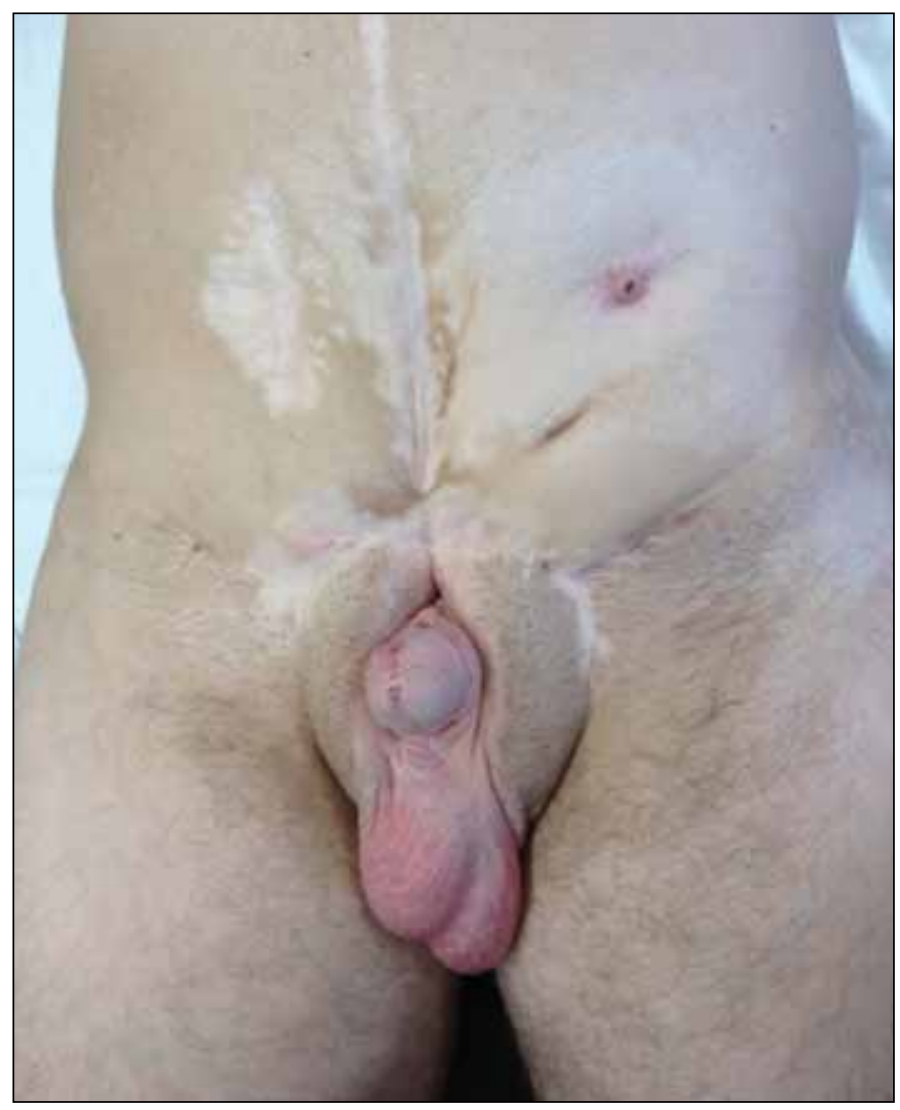

Fig. 4. Several complications may occur in a patient with a history extensive abdominal surgery and bladder exstrophy. A laparotomy is a high-risk undertaking in this patient. 
Valiquette et al.

surgery are major and could have profoundly altered the patient's quality of life. This case illustrates the importance of the CPS policy statement to provide developmentally appropriate care for youth as they move into an adult-oriented healthcare system.

Competing interests: Ms. Valiquette and Dr. Barrieras declare no competing financial or personal interests. Dr. McCormack is an Associate Editor at CUAJ.

This paper has been peer-reviewed.

\section{References}

1. Wiesner C, Bonfig R, Stein R, et al. Continent cutaneous urinary diversion: Long-term follow-up of more than 800 patients with ileocecal reservoirs. World J Urol 2006;24:315-8. http://dx.doi.org/10.1007/ s00345-006-0078-y

2. Holmes DG, Trasher JB, Park GY, et al. Long term complications related to the modified Indiana pouch. Urology 2002;60:603-6. http://dx.doi.org/10.1016/S0090-4295(02)01945-3

3. Rowland RG. Complications of continent cutaneous reservoirs and neobladders-series using contemporary techniques. AUA Update Series 1995; 14:202-7.

4. Webster C, Bukkapatnam R, Seigne JD. Continent colonic urinary reservoir (Florida pouch): Long term surgical complications (greater than 11 years). J Urol 2003;169:174-6. http://dx.doi.org/10.1016/ S0022-5347 (05)64061-X

5. Harris MA, Freeman KA, Duke DC. Transitioning from pediatric to adult health care: Dropping off the face of the earth. Am J Lifestyle Med 2011;5:85-91. http://dx.doi.org/10.1177/1559827610378343

6. Canadian Paediatric Society Adolescent Health Committee. Position statement: Transition to adult care for youth with special healthcare needs. Paediatr Child Health 2007;12:785-8.

Correspondence: Dr. Michael McCormack, Department of Surgery, University of Montreal, Montreal, QC; m.mccormack@umontreal.ca 